\title{
Early tumor shrinkage and depth of response in patients with advanced gastric cancer: a retrospective analysis of a randomized phase III study of first-line S-1 plus oxaliplatin vs. S-1 plus cisplatin
}

\author{
Tomohiro Nishina ${ }^{1} \cdot$ Mizutomo Azuma ${ }^{2} \cdot$ Kazuhiro Nishikawa ${ }^{3} \cdot$ Masahiro Gotoh $^{4} \cdot$ Hideaki Bando $^{5}$. \\ Naotoshi Sugimoto $^{6} \cdot$ Kenji Amagai $^{7} \cdot$ Keisho Chin $^{8} \cdot$ Yasumasa Niwa $^{9} \cdot$ Akihito Tsuji $^{10} \cdot$ Hiroshi Imamura $^{11}$. \\ Masahiro Tsuda $^{12}$. Hirofumi Yasui ${ }^{13} \cdot$ Hirofumi Fujii $^{14} \cdot$ Kensei Yamaguchi $^{15} \cdot$ Hisateru Yasui $^{16}$. Shuichi Hironaka ${ }^{17}$. \\ Ken Shimada ${ }^{18} \cdot$ Hiroto Miwa $^{19} \cdot$ Terukazu Mitome $^{20} \cdot$ Hiroki Kageyama $^{20} \cdot$ Ichinosuke Hyodo $^{21}$
}

Received: 30 March 2018 / Accepted: 1 June 2018 / Published online: 9 June 2018

(c) The International Gastric Cancer Association and The Japanese Gastric Cancer Association 2018

\begin{abstract}
Background We investigated early tumor shrinkage (ETS) and depth of response (DpR) using data from the G-SOX study comparing S-1 plus oxaliplatin with S-1 plus cisplatin as the first-line treatment for advanced gastric cancer (AGC).

Methods ETS was determined as \% decrease in the sum of the longest diameters of the target lesions at the first evaluation of week 6 compared to baseline. DpR was the maximum \% shrinkage during the study treatment. The impact of ETS (cutoff value 20\%) and DpR (continuous value) on progression-free survival (PFS) and overall survival (OS) were assessed by the log-rank test and Cox regression analysis including prognostic factors obtained in the G-SOX study; ECOG performance status, baseline sum of tumor diameters, disease status (recurrent/unresectable), and histology (diffuse/intestinal).

Results Among 685 patients enrolled in the G-SOX study, 632 patients who had the first tumor evaluation were analyzed. Patients with ETS $\geq 20 \%$ had longer PFS (median 4.5 vs. 2.8 months, $p<0.0001$ ) and OS (median 14.8 vs. 10.5 months, $p<0.0001$ ) than those with ETS $<20 \%$. Adjusted hazard ratios of ETS $<20$ vs. $\geq 20 \%$ were 0.606 (95\% confidence interval (CI) 0.506-0.725) for PFS and 0.589 (95\% CI 0.492-0.704) for OS. DpR was also significantly associated with PFS and OS (both $p<0.0001$ ). These results were similar between the SOX and CS groups.
\end{abstract}

Conclusions In AGC patients receiving the first-line therapy, ETS and DpR might be predictors for PFS and OS.

Keywords Gastric cancer · Early tumor shrinkage $\cdot$ Depth of response $\cdot$ Oxaliplatin $\cdot$ Chemotherapy

\section{Introduction}

One of the goals of chemotherapy for advanced gastric cancer (AGC) is to reduce the tumor size expecting amelioration of tumor-related symptoms and survival prolongation.

In the recent years, several retrospective studies [1-5] and one prospective study [6] have shown that early tumor shrinkage (ETS), defined as a \% decrease in the sum of the longest diameters of the target lesions at the first evaluation (usually week 6 or 8), is a good predictor of the long-term outcomes of the first-line treatment for metastatic colorectal cancer (mCRC). In most of these reports, ETS of $20 \%$ was

Tomohiro Nishina

tnishina@shikoku-cc.go.jp

Extended author information available on the last page of the article used to classify as responders or non-responders [2-6]. ETS is considered as an on-treatment marker that reflects tumor sensitivity to anticancer therapy $[2,7,8]$ and thus could be a useful clinical decision-making tool. In addition, depth of response (DpR) defined as the maximum tumor shrinkage of individual patient during the treatment has been reported to be associated with survival in $\mathrm{mCRC}$ patients $[5,6]$.

Unlike mCRC, there are a small number of studies assessing ETS or DpR in AGC [9-11]. Although those reports suggested that ETS was a significant predictor of the long-term outcomes in patients who received the combination therapy with trastuzumab against human epidermal growth factor receptor 2 (HER2), the association between ETS and treatment outcomes of the platform regimen, fluoropyrimidine plus platinum, is not known.

The phase III G-SOX study has demonstrated noninferiority of combination therapy with S-1 plus oxaliplatin 
(SOX) to cisplatin plus S-1 (CS) in the first-line treatment for AGC with regard to progression-free survival (PFS) and overall survival (OS) [12]. In this present study, we reviewed the G-SOX data to determine whether ETS and DpR are associated with the long-term outcomes in AGC patients.

\section{Patients and methods}

\section{G-SOX study}

The G-SOX study was a prospective, open-label, multicenter, randomized phase III clinical trial conducted at 51 centers in Japan. Between January 2010 and October 2011, 685 chemotherapy-naïve AGC patients with at least one measurable lesion at baseline were randomly assigned at a 1:1 ratio to receive first-line SOX or CS therapy, as reported previously [12]. Treatment was continued until disease progression, unacceptable toxicity, or patient's withdrawal. Tumors were monitored with computed tomography (CT) at baseline and every 6 weeks after randomization until the start of second-line therapy. All images were assessed by an independent review committee according to the Response Evaluation Criteria in Solid Tumors (RECIST) version 1.0 [13]. The G-SOX study was conducted in compliance with the Declaration of Helsinki and the Good Clinical Practice (GCP) guidelines, and the study protocol was approved by the ethical committee of each institution.

\section{Statistical analysis}

Patients with no CT scan data at week 6 were excluded from the full analysis set of the G-SOX study. All other patients were analyzed in the present study. ETS was assessed as the relative tumor reduction (\%) in the sum of the longest diameters of the target lesions at week 6 compared to the baseline. DpR was defined as the maximum tumor shrinkage (\%) observed throughout the treatment.

We compared baseline characteristics between two treatment groups by Fisher's exact test. Differences in response measurements were also compared. To validate combining two treatment groups for subsequent analyses, waterfall plots were created for each treatment group at the maximum shrinkage point. The time from randomization to maximum shrinkage was also calculated.

The landmark approach was used to avoid guarantee-time bias [14], and the first CT scan was set as the landmark point for ETS. PFS was defined as the time from the landmark point until disease progression or death from any cause (whichever was earlier). OS was defined as the time from the landmark point until death from any cause. Subpopulation treatment effect pattern plot (STEPP) analysis was performed to explore the interaction between treatment effects and ETS. A sliding-window approach (window size of 150 with an overlap between adjacent windows of 100 cases) was employed for the analysis $[15,16]$. We adopted the cutoff value of $20 \%$ for ETS recommended in various reports for mCRC [2-6]. Baseline characteristics were compared using Fisher's exact test between the patients who achieved ETS and patients who did not. PFS and OS curves were estimated by the Kaplan-Meier method for patients who achieved ETS $\geq 20 \%$ and patients who did not, and were compared by the log-rank test, respectively ( $\geq 20$ vs. $<20 \%$ ). To evaluate the impact of ETS or DpR on PFS and OS, hazard ratios (HRs) and their 95\% confidence intervals (CIs) were determined by multivariate Cox regression analysis. The multivariate analysis employed four prognostic factors [Eastern Cooperative Oncology Group performance status (ECOG PS; 1,2 vs. 0 ), total tumor diameter before the treatment ( $\geq$ median vs. $<$ median), disease status (recurrent vs. unresectable), and tumor histology (diffuse vs. intestinal)] that were reported to influence OS in the G-SOX study [12], along with the treatment (CS vs. SOX) and ETS or DpR.

To evaluate the predictive accuracy of ETS or DpR for PFS and OS, time-dependent receiver operating characteristics (ROC) analysis was performed to calculate the time-dependent concordance index $(\mathrm{C} \tau)$ [17], with which its $95 \%$ CI was calculated by the bootstrap method using 1000 samples, and as a reference, optimal cutoff values of ETS and DpR: the point on the ROC curve that was closest to the point at both sensitivity and specificity $=1$ (point of maximum sensitivity and specificity) were obtained [18].

Statistical significance level was set at $p<0.05$ (twosided). Due to the exploratory nature of this study, adjustment for multiplicity was not done during these analyses. All statistical analyses were performed by SAS version 9.4 (SAS Institute, Cary, NC, USA).

\section{Results}

\section{Patients}

G-SOX study enrolled a total of 685 patients. Of 685 randomized patients, twenty-two patients were not included in the full analysis set (9 did not receive the allocated treatment, 3 had a major GCP violation, and 10 did not meet the inclusion or exclusion criteria). Thirty-one patients with no tumor diameter data at the first tumor evaluation were also excluded, and remaining 632 patients (313 in the SOX group and 319 in the CS group) were included in the present study.

\section{Validity of combining two treatment groups}

Baseline characteristics were well balanced between the two treatment arms (Table 1). Median ETS was $23.5 \%$ in the 
Table 1 Baseline characteristics

\begin{tabular}{|c|c|c|c|}
\hline & \multicolumn{2}{|l|}{ Treatment } & \multirow[t]{2}{*}{$p^{\mathrm{a}}$} \\
\hline & $\operatorname{SOX}(N=313)$ & $\mathrm{CS}(N=319)$ & \\
\hline Age (years) & $66(21-83)$ & $65(29-85)$ & - \\
\hline \multicolumn{4}{|l|}{ Gender } \\
\hline Male & $235(75.1)$ & $235(73.7)$ & \multirow[t]{2}{*}{0.72} \\
\hline Female & $78(24.9)$ & $84(26.3)$ & \\
\hline \multicolumn{4}{|l|}{ ECOG performance status } \\
\hline 0 & $221(70.6)$ & $227(71.2)$ & \multirow[t]{3}{*}{0.98} \\
\hline 1 & $88(28.1)$ & $88(27.6)$ & \\
\hline 2 & $4(1.3)$ & $4(1.3)$ & \\
\hline Unresectable & $258(82.4)$ & $266(83.4)$ & \multirow[t]{4}{*}{0.75} \\
\hline Recurrent & $55(17.6)$ & $53(16.6)$ & \\
\hline Adjuvant chemotherapy (+) & $28(8.9)$ & $26(8.2)$ & \\
\hline Adjuvant chemotherapy (-) & $27(8.6)$ & $27(8.5)$ & \\
\hline \multicolumn{4}{|l|}{ Tumor histology } \\
\hline Intestinal & $145(46.3)$ & $143(44.8)$ & \multirow[t]{2}{*}{0.75} \\
\hline Diffuse & $168(53.7)$ & $176(55.2)$ & \\
\hline \multicolumn{4}{|l|}{ Primary tumor } \\
\hline- & $72(23.0)$ & $73(22.9)$ & \multirow[t]{2}{*}{1.0} \\
\hline+ & $241(77.0)$ & $246(77.1)$ & \\
\hline \multicolumn{4}{|l|}{ No. of metastatic sites } \\
\hline 1 & $100(31.9)$ & $101(31.7)$ & \multirow[t]{3}{*}{1.00} \\
\hline 2 & $134(42.8)$ & $137(42.9)$ & \\
\hline$\geq 3$ & $79(25.2)$ & $81(25.4)$ & \\
\hline \multicolumn{4}{|l|}{ Metastatic site ${ }^{\mathrm{b}}$} \\
\hline Liver & $123(39.3)$ & $127(39.8)$ & \multirow[t]{4}{*}{-} \\
\hline Lung & $35(11.2)$ & $33(10.3)$ & \\
\hline Lymph node & $286(91.4)$ & $282(88.4)$ & \\
\hline Peritoneal & $60(19.2)$ & 63 (19.7) & \\
\hline
\end{tabular}

Data are shown as the number (\%) or median (range)

SOX S-1 plus oxaliplatin, $C S$ cisplatin plus S-1, ECOG Eastern Cooperative Oncology Group

${ }^{\text {a}}$ Fisher's exact test

${ }^{\mathrm{b}}$ Some patients had more than one site

SOX group and $22.7 \%$ in the CS group (Table 2). ETS $\geq 20 \%$ was achieved in $58.1 \%$ of the SOX group and $55.8 \%$ of the CS group. The STEPP analysis showed that PFS rates at 4 months and OS rates at 12 months were similar between SOX and CS groups (Fig. 1a, c), and HRs of PFS and OS were near 1.0 for each subpopulation (Fig. 1b, d), where the horizontal axis represents median ETS of the patient subpopulations. The results of the analysis indicated that both SOX and CS have similar treatment effects across the value of ETS with respect to PFS and OS, suggesting that there was no interaction between treatment effects and ETS. Median DpR was $38.2 \%$ in the SOX group and $37.8 \%$ in the CS group (Table 2), and the distributions of tumor shrinkage were very similar between treatment arms (Fig. 2). The median time to maximum tumor shrinkage was 121 days in the SOX group and 120 days in the CS group. These data mentioned above indicated that ETS and DpR were very similar between SOX and CS groups, and could be analyzed together.

\section{Impact of ETS and DpR on survival}

Baseline characteristics between the patients with ETS $\geq 20 \%$ and ETS $<20 \%$ were similar, with the exception of a higher proportion of the patients who had primary tumor in the ETS $\geq 20 \%$ patients (Table 3). Comparing the patients who achieved ETS $\geq 20 \%$ to those with ETS $<20 \%$, PFS was significantly longer in the former group (4.5 vs. 2.8 months, HR $0.659,95 \%$ CI $0.552-0.787, p<0.0001$ ) and so was OS (14.8 vs. 10.5 months, HR $0.663,95 \%$ CI $0.556-0.791, p<0.0001$ ) (Fig. 3). No significant interactions between the treatment arms and ETS were seen for PFS $(p=0.231)$ and $\mathrm{OS}(p=0.382)$.

In multivariate Cox regression analyses (Table 4), ETS was significantly associated with PFS and OS irrespective of whether it was treated as a continuous or binary variable (cutoff value of 20\%). DpR was also significantly associated with PFS and OS.

\section{Predictive accuracy and optimal cutoff values of ETS and DpR}

ETS (continuous variable) and DpR moderately predicted PFS (C $\tau$ index $0.540,95 \%$ CI $0.561-0.612$ for ETS, and $0.697,95 \%$ CI $0.678-0.717$ for DpR) and OS (C $\tau$ index $0.569,95 \%$ CI $0.547-0.594$ for ETS, and $0.644,95 \%$ CI $0.622-0.663$ for DpR).

Optimal cutoff values of ETS and DpR for predicting the long-term outcomes were calculated on the basis of the median PFS and OS (4.14 and 12.78 months after the landmark point, respectively) by ROC analysis in all patients. The optimal ETS cutoff values to dichotomize the study population for predicting the prognosis were $19.9 \%$ for PFS and $20.0 \%$ for OS. The optimal DpR cutoff values were 36.7\% for PFS and $40.0 \%$ for OS.

\section{Discussion}

The present study revealed that ETS at week 6 and DpR were significantly associated with survival outcomes in patients receiving combination chemotherapy with S-1 plus a platinum agent as the first-line treatment for AGC. When adjusted with the treatment effect and prognostic factors, the risks of progression and death reduced by approximately $40 \%$ in patients with ETS $\geq 20 \%$, compared to those in patients with ETS $<20 \%$. ETS can be obtained at a specified early time point after the start of treatment, while the 
Table 2 Response measurements between treatment arms

\begin{tabular}{llll}
\hline & Treatment & & $p$ \\
\cline { 2 - 4 } & SOX $(N=313)(\%)$ & CS $(N=319)(\%)$ & \\
\hline RECIST response & & & $0.338^{\mathrm{a}}$ \\
Response rate (CR+PR) & 56.9 & 53.0 & \\
ETS & & & $0.769^{\mathrm{b}}$ \\
Median (range) & $23.5(-98.8$ to 65.6) & $22.7(-73.4$ to 100$)$ & $0.574^{\mathrm{a}}$ \\
ETS $\geq 20 \%$ & 58.1 & 55.8 & \\
ETS $<20 \%$ & 41.9 & 44.2 & $0.831^{\mathrm{b}}$ \\
DpR & & & $0.407^{\mathrm{a}}$ \\
Median (range) & $38.2(-98.8$ to 100) & $37.8(-73.4$ to 100$)$ & \\
1st quintile & 19.2 & 20.7 & \\
2nd quintile & 19.8 & 20.1 & 18.8 \\
3rd quintile & 21.7 & 17.9 & \\
4th quintile & 21.7 & 22.6 & \\
5th quintile & 17.6 & & \\
\hline
\end{tabular}

SOX oxaliplatin plus S-1, CS cisplatin plus S-1, RECIST Response Evaluation Criteria in Solid Tumors, $C R$ complete response, $P R$ partial response, ETS early tumor shrinkage, $D p R$ depth of response

${ }^{\text {a}}$ Fisher's exact test

${ }^{\mathrm{b}}$ Kruskal-Wallis test

time reaching the maximum shrinkage is longer and unpredictable. On this point, ETS is more useful than DpR as an on-treatment marker for predicting prognoses.

Several reports have demonstrated the correlation between ETS and survival in mCRC patients receiving chemotherapy with or without molecular-targeted agents, such as anti-EGFR monoclonal antibodies (cetuximab and panitumumab) and anti-VEGF monoclonal antibody (bevacizumab) [7]. The present study was based on the data of G-SOX study comparing SOX and CS without moleculartargeted agents. Trastuzumab was not approved for AGC in Japan when the G-SOX study was planned, and the HER2 status of the patients was not investigated. Our results indicated that ETS was applicable for survival prediction in AGC patients receiving the first-line chemotherapy without a molecular-targeted agent.

It is important to set an optimal cutoff value for appropriately classifying patients as responders and non-responders. The cutoff value for dichotomizing a patient population has been reported to depend on the regimen used for treatment $[4,6,7]$. In the present study, we used $20 \%$ cutoff value for
ETS based on the findings reported in mCRC studies [2-6]. By ROC analysis, we assured 20\% ETS as the optimal cutoff value for predicting PFS and OS in AGC patients treated with first-line chemotherapy with S-1 plus either cisplatin or oxaliplatin.

In the absence of pretreatment predictors of response, a regular evaluation of tumor shrinkage is usually performed on the basis of the RECIST and used for the decision-making of treatment continuation and prediction of prognosis. In the RECIST, 30\% tumor shrinkage is a cutoff value for responder and non-responder [13]. The time point reaching the cutoff is not defined. Although we did not analyze the relationship between ETS and RECIST responders, ETS at week 6 seemed to be simple and easy to use for treating physicians.

In conclusion, both ETS and DpR were associated with the long-term outcomes in AGC patients receiving first-line SOX or CS therapy. In particular, $20 \%$ tumor shrinkage at week 6 could be applicable as the cutoff value for predicting survival. The present analyses were retrospective using a single study, and further studies are needed for verification. 

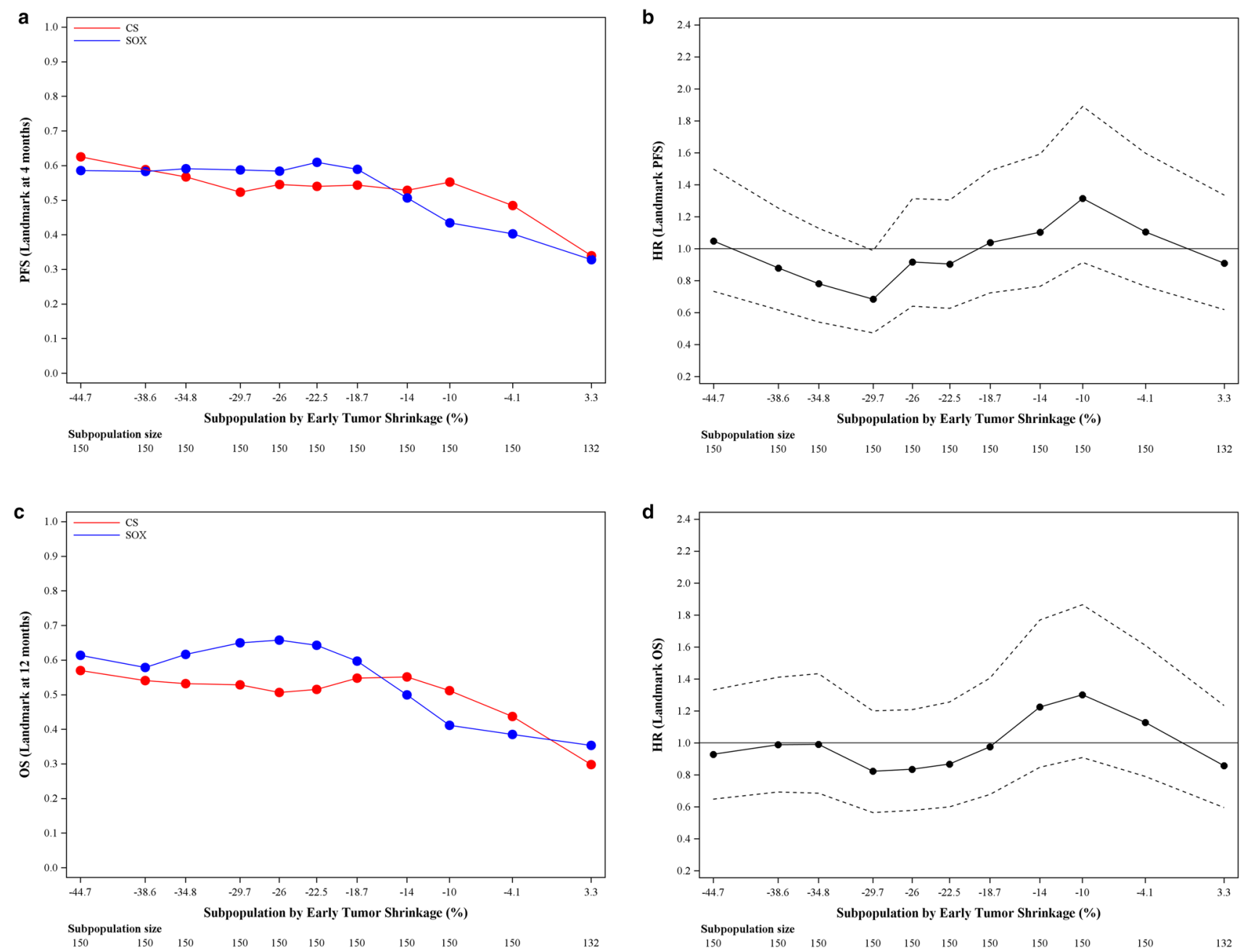

Fig. 1 Subpopulation treatment effect pattern plot analysis of a progression-free survival (PFS) at 4 months from landmark, b hazard ratio (HR) of PFS, $\mathbf{c}$ overall survival (OS) at 12 months from landmark, and $\mathbf{d}$ HR of OS
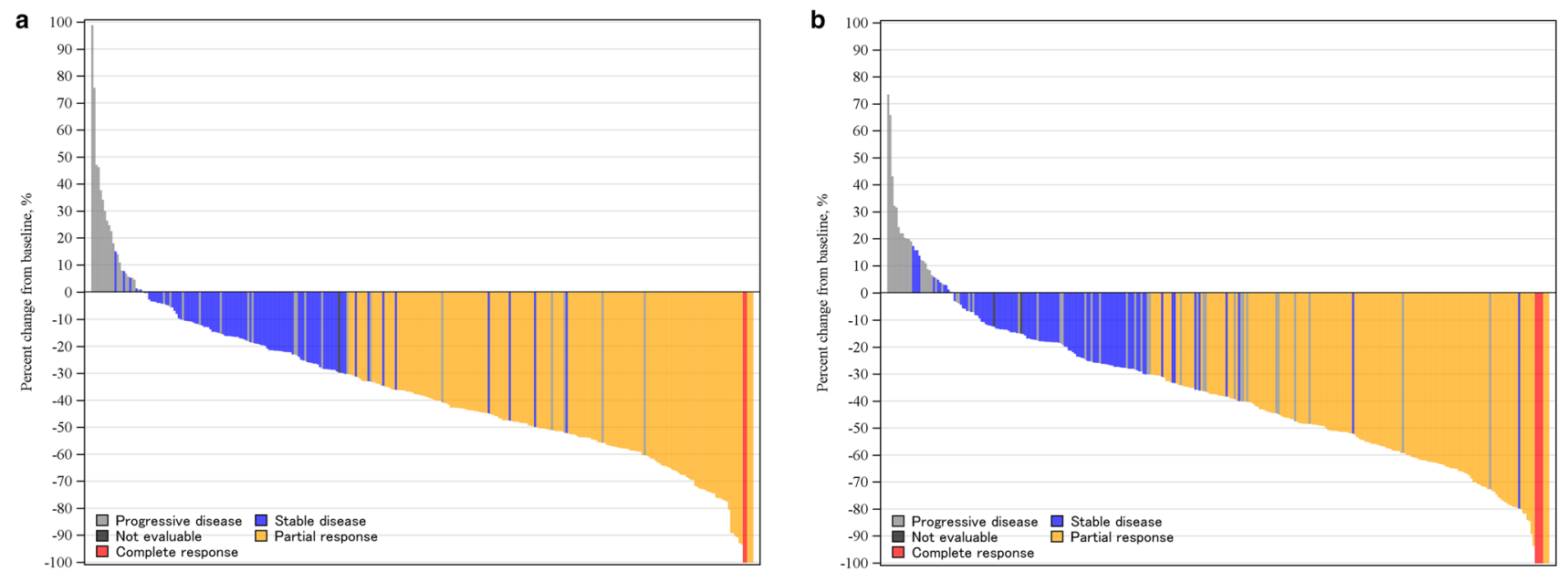

Fig. 2 Depth of response in both groups, a SOX group; b CS group 
Table 3 Baseline characteristics based on ETS $<20$ vs. $\geq 20 \%$

\begin{tabular}{|c|c|c|c|}
\hline & \multicolumn{2}{|c|}{ Early tumor shrinkage } & \multirow[t]{2}{*}{$p^{\mathrm{a}}$} \\
\hline & $<20 \%(N=272)$ & $\geq 20 \%(N=360)$ & \\
\hline Age (years) & $65(21-83)$ & $65(31-85)$ & - \\
\hline \multicolumn{4}{|l|}{ Gender } \\
\hline Male & $202(74.3)$ & $268(74.4)$ & \multirow[t]{2}{*}{1.00} \\
\hline Female & $70(25.7)$ & $92(25.6)$ & \\
\hline \multicolumn{4}{|l|}{ ECOG performance status } \\
\hline 0 & $193(71.0)$ & $255(70.8)$ & \multirow[t]{3}{*}{1.00} \\
\hline 1 & $76(27.9)$ & $100(27.8)$ & \\
\hline 2 & $3(1.1)$ & $5(1.4)$ & \\
\hline Unresectable & $217(79.8)$ & $307(85.3)$ & \multirow[t]{4}{*}{0.07} \\
\hline Recurrent & $55(20.2)$ & $53(14.7)$ & \\
\hline Adjuvant chemotherapy (+) & $30(11.0)$ & $24(6.7)$ & \\
\hline Adjuvant chemotherapy (-) & $25(9.2)$ & $29(8.1)$ & \\
\hline \multicolumn{4}{|l|}{ Tumor histology } \\
\hline Intestinal & $133(48.9)$ & $155(43.1)$ & \multirow[t]{2}{*}{0.15} \\
\hline Diffuse & $139(51.1)$ & $205(56.9)$ & \\
\hline \multicolumn{4}{|l|}{ Primary tumor } \\
\hline- & $74(27.2)$ & $71(19.7)$ & \multirow[t]{2}{*}{0.03} \\
\hline+ & $198(72.8)$ & $289(80.3)$ & \\
\hline \multicolumn{4}{|l|}{ No. of metastatic sites } \\
\hline 1 & $77(28.3)$ & $124(34.4)$ & \multirow[t]{3}{*}{0.18} \\
\hline 2 & $127(46.7)$ & $144(40.0)$ & \\
\hline$\geq 3$ & $68(25.0)$ & $92(25.6)$ & \\
\hline \multicolumn{4}{|l|}{ Metastatic site ${ }^{\mathrm{b}}$} \\
\hline Liver & 117 (43.0) & 133 (36.9) & \multirow[t]{4}{*}{-} \\
\hline Lung & $32(11.8)$ & $36(10.0)$ & \\
\hline Lymph node & $242(89.0)$ & $326(90.6)$ & \\
\hline Peritoneal & $57(21.0)$ & $66(18.3)$ & \\
\hline
\end{tabular}

Data are shown as the number (\%) or median (range)

ECOG Eastern Cooperative Oncology Group

${ }^{\mathrm{a}}$ Fisher's exact test

${ }^{\mathrm{b}}$ Some patients had more than one site 

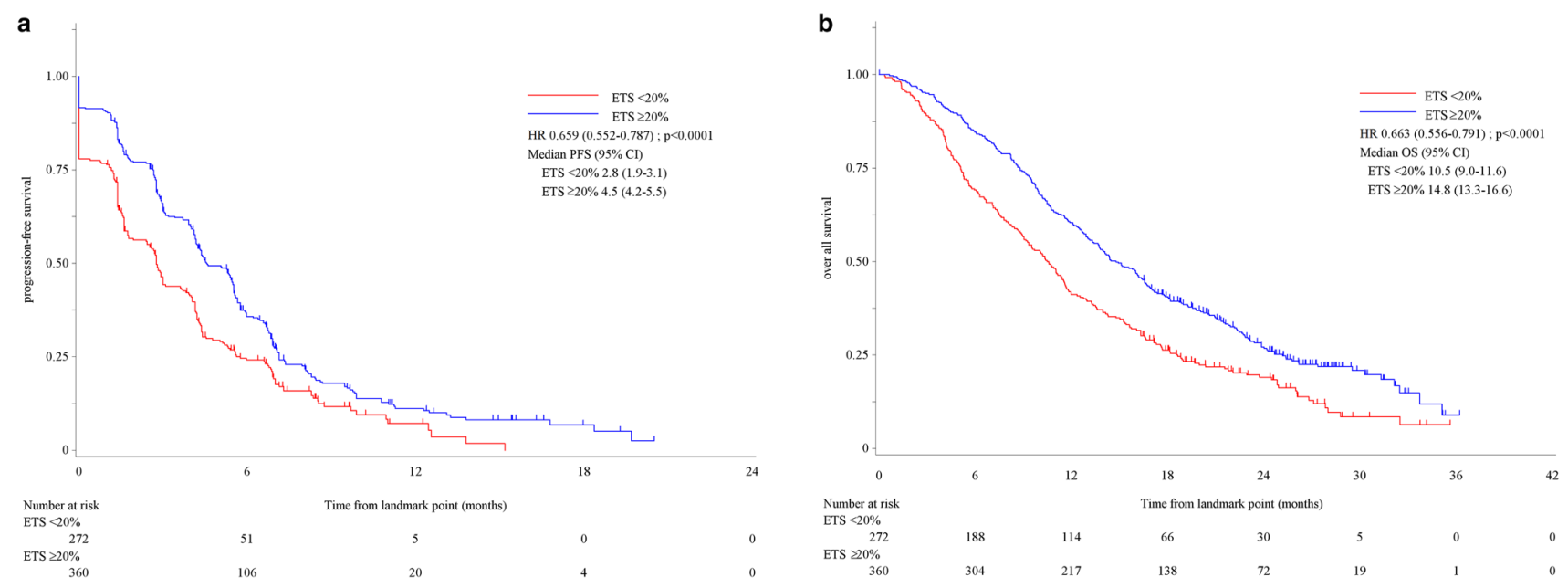

Fig. 3 Kaplan-Meier survival curves stratified according to tumor shrinkage ( $<20$ vs. $\geq 20 \%)$ at first evaluation, a progression-free survival (PFS); b overall survival (OS)

Table 4 Impact of ETS or DpR on PFS and OS

\begin{tabular}{|c|c|c|c|c|c|c|c|c|c|}
\hline \multirow[t]{2}{*}{ Variables } & \multirow[t]{2}{*}{ Category } & \multicolumn{4}{|l|}{ PFS } & \multicolumn{4}{|l|}{ OS } \\
\hline & & HR & $95 \% \mathrm{CI}$ & & $p^{\mathrm{a}}$ & HR & $95 \% \mathrm{CI}$ & & $p^{\mathrm{a}}$ \\
\hline ETS as continuous ${ }^{\mathrm{b}}$ & & 0.808 & 0.767 & 0.851 & $<0.0001$ & 0.851 & 0.815 & 0.889 & $<0.0001$ \\
\hline Treatment & SOX (vs. CS) & 0.952 & 0.798 & 1.137 & 0.5893 & 0.991 & 0.831 & 1.182 & 0.9223 \\
\hline ECOG PS & 1,2 (vs. 0) & 1.434 & 1.185 & 1.735 & 0.0002 & 1.662 & 1.374 & 2.010 & $<0.0001$ \\
\hline Disease status & Recurrent (vs. unresectable) & 0.663 & 0.512 & 0.858 & 0.0018 & 0.509 & 0.388 & 0.667 & $<0.0001$ \\
\hline Tumor histology & Diffuse (vs. intestinal) & 1.385 & 1.157 & 1.659 & 0.0004 & 1.491 & 1.247 & 1.784 & $<0.0001$ \\
\hline Sum of tumor diameter & $\geq \operatorname{Median}^{\mathrm{c}}($ vs $<$ median $)$ & 1.511 & 1.258 & 1.815 & $<0.0001$ & 1.493 & 1.246 & 1.789 & $<0.0001$ \\
\hline ETS & $\geq 20 \%$ (vs. $<20 \%$ ) & 0.606 & 0.506 & 0.725 & $<0.0001$ & 0.589 & 0.492 & 0.704 & $<0.0001$ \\
\hline Treatment & SOX (vs. CS) & 0.962 & 0.806 & 1.148 & 0.6686 & 0.989 & 0.830 & 1.180 & 0.9059 \\
\hline ECOG PS & 1,2 (vs. 0) & 1.433 & 1.185 & 1.734 & 0.0002 & 1.657 & 1.370 & 2.004 & $<0.0001$ \\
\hline Disease status & Recurrent (vs. unresectable) & 0.701 & 0.543 & 0.905 & 0.0065 & 0.550 & 0.422 & 0.718 & $<0.0001$ \\
\hline Tumor histology & Diffuse (vs. intestinal) & 1.291 & 1.080 & 1.544 & 0.0050 & 1.474 & 1.232 & 1.765 & $<0.0001$ \\
\hline Sum of tumor diameter & $\geq \operatorname{Median}^{\mathrm{c}}(\mathrm{vs}<$ median $)$ & 1.493 & 1.244 & 1.793 & $<0.0001$ & 1.495 & 1.249 & 1.791 & $<0.0001$ \\
\hline $\mathrm{DpR}$ as continuous ${ }^{\mathrm{b}}$ & & 0.741 & 0.712 & 0.771 & $<0.0001$ & 0.829 & 0.803 & 0.856 & $<0.0001$ \\
\hline Treatment & SOX (vs. CS) & 0.916 & 0.767 & 1.094 & 0.3334 & 0.977 & 0.820 & 1.165 & 0.7986 \\
\hline ECOG PS & 1,2 (vs. 0) & 1.360 & 1.124 & 1.646 & 0.0016 & 1.648 & 1.363 & 1.993 & $<0.0001$ \\
\hline Disease status & Recurrent (vs. unresectable) & 0.622 & 0.480 & 0.807 & 0.0004 & 0.466 & 0.354 & 0.613 & $<0.0001$ \\
\hline Tumor histology & Diffuse (vs. intestinal) & 1.262 & 1.056 & 1.508 & 0.0104 & 1.417 & 1.186 & 1.693 & 0.0001 \\
\hline Sum of tumor diameter & $\geq \operatorname{Median}^{\mathrm{c}}($ vs $<$ median $)$ & 1.487 & 1.236 & 1.789 & $<0.0001$ & 1.441 & 1.202 & 1.726 & $<0.0001$ \\
\hline
\end{tabular}

$P F S$ progression-free survival, $O S$ overall survival, $H R$ hazard ratio, $C I$ confidence interval, $C S$ cisplatin plus $\mathrm{S}-1, S O X$ oxaliplatin plus $\mathrm{S}-1$, ECOG Eastern Cooperative Oncology Group, $P S$ performance status, ETS early tumor shrinkage, $D p R$ depth of response

${ }^{\mathrm{a}}$ Wald test

${ }^{\mathrm{b}} \mathrm{HRs}$ are presented per $10 \%$ tumor shrinkage

${ }^{\mathrm{c}}$ Median sum of tumor diameters: $77.6 \mathrm{~mm}$

Acknowledgements This work was supported by Yakult Honsha. We would like to thank all of the patients, investigators, and support staff who participated in the G-SOX study. We are also grateful to Atsushi Sato, Kunihisa Miyakawa, Tohru Fukushima, Tsuyoshi Morimoto, and Shinjiro Sakaino for performing extramural review to assess the objective response and PFS. We sincerely acknowledge the contribution of the late Chikuma Hamada, the former statistical advisor of the G-SOX study. 


\section{Compliance with ethical standards}

Conflict of interest Tomohiro Nishina has received honoraria from Yakult Honsha and Taiho Pharmaceutical. Kazuhiro Nishikawa has received grants and personal fees from Yakult Honsha, personal fees from Taiho Pharmaceutical, Chugai Pharmaceutical, Eli Lilly and EA Pharma. Masahiro Gotoh has received grants, personal fees and nonfinancial support from Taiho Pharmaceutical, non-financial support from Yakult Honsha, personal fees and non-financial support from Bristol-Myers Squibb, Chugai Pharmaceutical, Takeda Pharmaceutical, Kyowa Hakko Kirin, Novartis Pharmaceutical, Sumitomo Dainippon Pharma, Bayer and Ono Pharmaceutical. Hideaki Bando has received research funds from Astra-Zeneca and Chugai Pharmaceutical, honoraria from Taiho Pharmaceutical and Eli Lilly. Naotoshi Sugimoto has received honoraria from Yakult Honsha, Taiho Pharmaceutical, Eli Lilly, Merck Serono and Chugai Pharmaceutical. Kenji Amagai has received research funding from Taiho Pharmaceutical and MSD. Akihito Tsuji has received honoraria from Yakult Honsha and Taiho Pharmaceutical. Kensei Yamaguchi has received personal fees from Taiho Pharmaceutical, Bayer, Bristol-Myers Squibb, Merck Serono, Chugai Pharmaceutical and Takeda Pharmaceutical. Hisateru Yasui has received personal fees from Medicon. Hisateru Yasui has received honoraria from Yakult Honsha, Taiho Pharmaceutical, Chugai Pharmaceutical, and Medicon Inc. Shuichi Hironaka has received honoraria from Yakult Honsha, Taiho Pharmaceutical, and Novartis Pharmaceutical. Ken Shimada has received honoraria from Yakult Honsha, Taiho Pharmaceutical, Chugai Pharmaceutical and Kyowa Hakko Kirin. Terukazu Mitome and Hiroki Kageyama are employees of Yakult Honsha, which produced oxaliplatin used in the G-SOX study from which data were obtained for the present analysis. Ichinosuke Hyodo has received advisory fees and honoraria from Yakult Honsha, Taiho Pharmaceutical and Chugai Pharmaceutical. The other authors have declared no conflicts of interest.

Human rights statement and informed consent All procedures followed were in accordance with the ethical standards of the responsible committees on human experimentation (institutional and national) and with the Helsinki Declaration of 1964 and later versions. Informed consent or a substitute for it was obtained from all patients for being included in the study.

\section{References}

1. Suzuki C, Blomqvist L, Sundin A, Jacobsson H, Byström P, Berglund $\AA$, et al. The initial change in tumor size predicts response and survival in patients with metastatic colorectal cancer treated with combination chemotherapy. Ann Oncol. 2012;23(4):948-54.

2. Giessen C, Laubender RP, Fischer von Weikersthal L, Schalhorn A, Modest DP, Stintzing S, et al. Early tumor shrinkage in metastatic colorectal cancer: retrospective analysis from an irinotecanbased randomized first-line trial. Cancer Sci. 2013;104(6):718-24.

3. Piessevaux H, Buyse M, Schlichting M, Van Cutsem E, Bokemeyer $\mathrm{C}$, Heeger $\mathrm{S}$, et al. Use of early tumor shrinkage to predict long-term outcome in metastatic colorectal cancer treated with cetuximab. J Clin Oncol. 2013;31(30):3764-75.

4. Modest DP, Laubender RP, Stintzing S, Giessen C, Schulz C, Haas M, et al. Early tumor shrinkage in patients with metastatic colorectal cancer receiving first-line treatment with cetuximab combined with either CAPIRI or CAPOX: an analysis of the German AIO KRK 0104 trial. Acta Oncol. 2013;52(5):956-62.

5. Cremolini C, Loupakis F, Antoniotti C, Lonardi S, Masi G, Salvatore $\mathrm{L}$, et al. Early tumor shrinkage and depth of response predict long-term outcome in metastatic colorectal cancer patients treated with first-line chemotherapy plus bevacizumab: results from phase III TRIBE trial by the Gruppo Oncologico del Nord Ovest. Ann Oncol. 2015;26(6):1188-94.

6. Tsuji A, Sunakawa Y, Ichikawa W, Nakamura M, Kochi M, Denda $\mathrm{T}$, et al. Early tumor shrinkage and depth of response as predictors of favorable treatment outcomes in patients with metastatic colorectal cancer treated with FOLFOX plus cetuximab (JACCRO CC-05). Target Oncol. 2016;11(6):799-806.

7. Heinemann V, Stintzing S, Modest DP, Giessen-Jung C, Michl M, Mansmann UR. Early tumour shrinkage (ETS) and depth of response $(\mathrm{DpR})$ in the treatment of patients with metastatic colorectal cancer (mCRC). Eur J Cancer. 2015;51(14):1927-36.

8. Modest DP, Stintzing S, Fischer von Weikersthal L, Decker T, Kiani A, Vehling-Kaiser U, et al. Relation of early tumor shrinkage (ETS) observed in first-line treatment to efficacy parameters of subsequent treatment in FIRE-3 (AIOKRK0306). Int J Cancer 2017;140(8):1918-25.

9. Osumi H, Takahari D, Shinozaki E, Chin K, Ogura M, Wakatsuki $\mathrm{T}$, et al. Associations between early tumor shrinkage and depth of response and clinical outcomes in patients treated with 1stline chemotherapy for advanced gastric cancer. Gastric Cancer. 2018;21(2):267-75.

10. Lee CK, Kim SS, Park S, Kim C, Heo SJ, Lim JS, et al. Depth of response is a significant predictor for long-term outcome in advanced gastric cancer patients treated with trastuzumab. Oncotarget. 2017;8:31169-79.

11. Kadowaki S, Masuishi T, Eto T, Narita Y, Taniguchi H, Ura T, et al. Depth of response predicts the clinical outcome of advanced HER2-positive gastric cancer to trastuzumab-based first-line chemotherapy. Cancer Chemother Pharmacol. 2017;80(4):807-13.

12. Yamada Y, Higuchi K, Nishikawa K, Gotoh M, Fuse N, Sugimoto N, et al. Phase III study comparing oxaliplatin plus S-1 with cisplatin plus S-1 in chemotherapy-naïve patients with advanced gastric cancer. Ann Oncol. 2015;26(1):141-8.

13. Therasse P, Arbuck SG, Eisenhauer EA, Wanders J, Kaplan RS, Rubinstein $\mathrm{L}$, et al. New guidelines to evaluate the response to treatment in solid tumors. European Organization for Research and Treatment of Cancer, National Cancer Institute of the United States, National Cancer Institute of Canada. J Natl Cancer Inst. 2000;92(3):205-16.

14. Giobbie-Hurder A, Gelber RD, Regan MM. Challenges of guarantee-time bias. J Clin Oncol. 2013;31(23):2963-69.

15. Bonetti M, Gelber RD. A graphical method to assess treatmentcovariate interactions using the Cox model on subsets of the data. Stat Med. 2000;19:2595-609.

16. Lazar AA, Cole BF, Bonetti M, et al. Evaluation of treatmenteffect heterogeneity using biomarkers measured on a continuous scale: subpopulation treatment effect pattern plot. J Clin Oncol. 2010;28(29):4539-44.

17. Uno H, Cai T, Pencina MJ, D’Agostino RB, Wei LJ. On the $\mathrm{C}$-statistics for evaluating overall adequacy of risk prediction procedures with censored survival data. Stat Med. 2011;30(10):1105-17.

18. Kumar R, Indrayan A. Receiver operating characteristic (ROC) curve for medical researchers. Indian Pediatr. 2011;48(4):277-87. 


\section{Affiliations}

Tomohiro Nishina $^{1} \cdot$ Mizutomo Azuma $^{2} \cdot$ Kazuhiro Nishikawa $^{3} \cdot$ Masahiro Gotoh $^{4} \cdot$ Hideaki Bando $^{5}$. Naotoshi Sugimoto ${ }^{6} \cdot$ Kenji Amagai $^{7} \cdot$ Keisho Chin $^{8}$. Yasumasa Niwa ${ }^{9} \cdot$ Akihito Tsuji $^{10}$. Hiroshi Imamura ${ }^{11}$. Masahiro Tsuda ${ }^{12}$. Hirofumi Yasui ${ }^{13} \cdot$ Hirofumi Fujii $^{14} \cdot$ Kensei Yamaguchi $^{15} \cdot$ Hisateru Yasui ${ }^{16}$. Shuichi Hironaka ${ }^{17}$. Ken Shimada ${ }^{18} \cdot$ Hiroto Miwa $^{19} \cdot$ Terukazu Mitome $^{20} \cdot$ Hiroki Kageyama $^{20} \cdot$ Ichinosuke Hyodo $^{21}$

1 Department of Gastrointestinal Medical Oncology, National Hospital Organization Shikoku Cancer Center, $160 \mathrm{Kou,}$ Minamiumemotomachi, Matsuyama 791-0280, Japan

2 Department of Gastroenterology, Kitasato University East Hospital, 2-1-1 Asamizodai, Minami-ku, Sagamihara 252-0380, Japan

3 Department of Surgery, National Hospital Organization Osaka National Hospital, 2-1-14 Hoenzaka, Chuo-ku, Osaka 540-0006, Japan

4 Cancer Chemotherapy Center, Osaka Medical College Hospital, 2-7 Daigakumachi, Takatsuki 569-8686, Japan

5 Division of Gastrointestinal Oncology and Digestive Endoscopy, National Cancer Center Hospital East, 6-5-1 Kashiwanoha, Kashiwa 277-0882, Japan

6 Department of Clinical Oncology, Osaka Medical Center for Cancer and Cardiovascular Diseases, 1-3-3 Nakamichi, Higashinari-ku, Osaka 537-8511, Japan

7 Department of Gastroenterology, Ibaraki Prefectural Central Hospital, 6528 Koibuchi, Kasama 309-1703, Japan

8 Department of Gastroenterology, Cancer Institute Hospital of JFCR, 3-8-31 Ariake, Tokyo 135-8550, Japan

9 Department of Endoscopy, Aichi Cancer Center Hospital, 1-1 Kanokoden, Chikusa-ku, Nagoya 464-8681, Japan

10 Department of Medical Oncology, Kochi Health Sciences Center, 2125-1 Ike, Kochi 781-8555, Japan

11 Department of Surgery, Sakai City Hospital, 1-1-1 Minamiyasui-cho, Sakai 590-0064, Japan
12 Department of Gastroenterological Oncology, Hyogo Cancer Center, 13-70 Kitaoji-cho, Akashi 673-8558, Japan

13 Division of Gastrointestinal Oncology, Shizuoka Cancer Center, 1007 Nagaizumi-cho, Shimonagakubo, Sunto-gun 411-8777, Japan

14 Department of Clinical Oncology, Jichi Medical University, 3311-1 Yakushiji, Shimotsuke 329-0498, Japan

15 Division of Gastroenterology, Saitama Cancer Center, 780 Inamachi Oaza Komuro, Kita-adachi-gun 362-0806, Japan

16 Department of Medical Oncology, National Hospital Organization Kyoto Medical Center, 1-1 Fukakusamukaihata-cho, Fushimi-ku, Kyoto 612-0861, Japan

17 Clinical Trial Promotion Department, Chiba Cancer Center, 666-2 Nitona-cho, Chuo-ku, Chiba 260-8717, Japan

18 Department of Internal Medicine, Showa University Northern Yokohama Hospital, Chigasakichuo, Tsuzuki-ku, Yokohama 224-0032, Japan

19 Division of Gastroenterology, Department of Internal Medicine, Hyogo College of Medicine, 1-1 Mukogawa-cho, Nishinomiya 663-8131, Japan

20 Pharmaceutical Research and Development Department, Yakult Honsha Co., Ltd., 16-21 Ginza 7-chome, Chuo-ku, Tokyo 104-0061, Japan

21 Division of Gastroenterology, University of Tsukuba, 1-1-1 Tennodai, Tsukuba 305-8575, Japan 